\title{
QUOT FUNCTORS FOR DELIGNE-MUMFORD STACKS
}

\author{
MARTIN OLSSON AND JASON STARR
}

Department of Mathematics, Massachusetts Institute of Technology, Cambridge, MA 02139, molsson@math.mit.edu

Department of Mathematics, Massachusetts Institute of Technology, Cambridge, MA 02139, jstarr@math.mit.edu

Dedicated to S. Kleiman on the occasion of his 60th birthday

\begin{abstract}
Given a separated and locally finitely-presented DeligneMumford stack $\mathcal{X}$ over an algebraic space $S$, and a locally finitelypresented $\mathcal{O}_{\mathcal{X}}$-module $\mathcal{F}$, we prove that the Quot functor $\operatorname{Quot}(\mathcal{F} / \mathcal{X} / S)$ is represented by a separated and locally finitely-presented algebraic space over $S$. Under additional hypotheses, we prove that the connected components of $\operatorname{Quot}(\mathcal{F} / \mathcal{X} / S)$ are quasi-projective over $S$.
\end{abstract}

\section{Contents}

1. Statement of results

2. Representability by an algebraic space

3. Flattening stratifications

4. Hilbert polynomials

4. Hilbert polynomials 13

5. Generating sheaves 15

6. Natural transformation of Quot functors 21

References 23

\section{Statement of Results}

Let $p: \mathcal{X} \rightarrow S$ be a separated, locally finitely-presented 1-morphism from a Deligne-Mumford stack $\mathcal{X}$ to an algebraic space $S$. Let $\mathcal{F}$ be a quasi-coherent $\mathcal{O}_{\mathcal{X}}$-module (on the étale site of $\mathcal{X}$ ) such that $\mathcal{F}$ is locally finitely-presented. Define a contravariant functor

$$
Q=Q(\mathcal{F} / \mathcal{X} / S): S-\text { schemes } \rightarrow \text { Sets }
$$

as follows. For each $S$-scheme $f: Z \rightarrow S$, define $\mathcal{X}_{Z}$ to be $\mathcal{X} \times{ }_{S} Z$, and define $\mathcal{F}_{Z}$ to be the pullback of $\mathcal{F}$ to $\mathcal{X}_{Z}$. We define $Q(Z)$ to be the set of $\mathcal{O}_{\mathcal{X}_{Z}}$-module quotients $\mathcal{F}_{Z} \rightarrow G$ which satisfy 
(1) $G$ is a quasi-coherent $\mathcal{O}_{\mathcal{X}_{Z}}$-module which is locally finitely-presented,

(2) $G$ is flat over $Z$,

(3) the support of $G$ is proper over $Z$.

All of these properties are preserved by base-change on $Z$, and therefore pullback makes $Q$ into a contravariant functor.

Theorem 1.1. $Q$ is represented by an algebraic space which is separated and locally finitely-presented over $S$. If $\mathcal{F}$ has proper support over $S$, then $Q \rightarrow S$ satisfies the valuative criterion for properness.

Remark: Under the hypothesis that $\mathcal{F}$ has proper support over $S$, we are not claiming that $Q \rightarrow S$ is proper, because we do not show that $Q \rightarrow S$ is quasi-compact (in general it need not be).

We give a better description of $Q$ under additional hypotheses on $\mathcal{X}$. Our first hypothesis is that $\mathcal{X}$ is a global quotient.

Definition 1.2. Let $S$ be an algebraic space. A global quotient stack over $S$ is an (Artin) algebraic stack $\mathcal{X}$ over $S$ which is isomorphic to a stack of the form $[Z / G]$, where $Z$ is an algebraic space which is finitely-presented over $S$, and $G$ is a flat, finitely-presented group scheme over $S$ which is a subgroup scheme of the general linear group scheme $G L_{n, S}$ for some $n$.

This is essentially [5, definition 2.9] (with the Noetherian hypotheses replaced by a finite-presentation hypothesis). We remind the reader of the following characterization in [5, remark 2.11].

Lemma 1.3. Suppose that $\mathcal{X}$ is a global quotient stack over $S$ which is isomorphic to $[Z / G]$ as above. Then the diagonal action of $G$ on $Z \times_{S} G L_{n, S}$ is free and the quotient $Z^{\prime}=\left[Z \times{ }_{S} G L_{n, S} / G\right]$ is an algebraic space with a right action of $G L_{n, S}$. The quotient stack $\left[Z^{\prime} / G L_{n, S}\right]$ is isomorphic to the original stack $[Z / G]$.

So every quotient stack is isomorphic to a stack of the form $\left[Z^{\prime} / G L_{n, S}\right]$.

Our second hypothesis is that $\mathcal{X}$ is a tame Deligne-Mumford stack.

Definition 1.4. A Deligne-Mumford stack $\mathcal{X}$ is tame if for any algebraically closed field $k$ and any 1-morphism $\zeta:$ Spec $k \rightarrow \mathcal{X}$, the stabilizer group Aut $(\zeta)($ Spec $k)$ has order prime to char $(k)$, where Aut $(\zeta)$ is the finite $k$-group scheme defined to be the Cartesian product of the diagram

$$
\begin{gathered}
\text { Spec } k \\
\\
\\
\downarrow \\
\mathcal{X} \stackrel{\Delta(\zeta, \zeta)}{\longrightarrow} \mathcal{X} \times \mathcal{X} .
\end{gathered}
$$

Here $\Delta: \mathcal{X} \rightarrow \mathcal{X} \times \mathcal{X}$ is the diagonal morphism.

Theorem 1.5. Suppose that $S$ is an affine scheme, and let $f: \mathcal{X} \rightarrow S$ be a separated 1-morphism from a tame Deligne-Mumford stack to $S$ such that $\mathcal{X}$ is a global quotient over $S$ and such that the coarse moduli space $X$ 
of $\mathcal{X}$ is a quasi-projective $S$-scheme (resp. projective $S$-scheme). Then the connected components of $Q$ are quasi-projective $S$-schemes (resp. projective S-schemes).

Remark: The existence of the coarse moduli space for $\mathcal{X}$ follows from [7.

Remark: The condition that $S$ be an affine scheme is required because the property of being quasi-projective is not Zariski local on the base.

Acknowledgments: The authors benefitted from useful conversations with Johan de Jong. The authors also wish to thank the referee, particularly for his suggestion to simplify section 5 by including theorem 5.2 .

This work was largely done during the semester program on Algebraic Stacks, Intersection Theory and Non-Abelian Hodge Theory at MSRI during the spring of 2002 where both authors were MSRI Postdoctoral Fellows. The first author was partially supported by an NSF Postdoctoral Fellowship. The second author was partially supported by NSF grant DMS-0201423.

\section{Representability By AN ALGEBRAiC SPACE}

In this section we prove theorem 1.1

Note first that $Q$ is a sheaf for the fppf-topology by descent theory, and is limit preserving. In addition, for each open substack $\mathcal{U} \subset \mathcal{X}$ there is a natural open immersion $Q\left(\left.\mathcal{F}\right|_{\mathcal{U}} / \mathcal{U} / S\right) \subset Q$. Moreover, $Q$ is the union over finitely presented open substacks $\mathcal{U} \subset \mathcal{X}$ of the $Q(\mathcal{F} \mid \mathcal{U} / \mathcal{U} / S)$. We may therefore assume that $\mathcal{X}$ is of finite presentation over $S$. Since the question of representability of $Q$ is étale local on $S$ we may assume that $S$ is an affine scheme, and by a standard limit argument we may assume that $S$ is of finite type over $\operatorname{Spec}(\mathbb{Z})$.

Under these assumptions we prove the theorem by verifying the conditions of theorem 5.3 of $[2]$.

Commutation with inverse limits. We need the Grothendieck existence theorem for Deligne-Mumford stacks:

Proposition 2.1. Let $A$ be a complete noetherian local ring, $\mathcal{X} / A$ a DeligneMumford stack of finite type, and let $A_{n}=A / \mathfrak{m}_{A}^{n+1}, \mathcal{X}_{n}=\mathcal{X} \otimes_{A} A_{n}$. Then the natural functor

\section{(coherent sheaves on $\mathcal{X}$ with support proper over $A$ )}

(compatible families of coherent sheaves on the $\mathcal{X}_{n}$ with proper support)

is an equivalence of categories. 
Proof. This proposition is perhaps best viewed in a context of formal algebraic stacks, but since we do not want to develop such a theory here we take a more ad-hoc approach.

Let $\widehat{\mathcal{X}}$ be the ringed topos $\left(\mathcal{X}_{0, e t}, \mathcal{O}_{\widehat{\mathcal{X}}}\right)$, where $\mathcal{O}_{\widehat{\mathcal{X}}}$ is the sheaf of rings which to any étale $\mathcal{X}_{0}$-scheme $U_{0} \rightarrow \mathcal{X}_{0}$ associates

$$
\lim _{\longleftarrow} \Gamma\left(U_{n}, \mathcal{O}_{U_{n}}\right) \text {. }
$$

Here $U_{n}$ denotes the unique lifting of $U_{0}$ to an étale $\mathcal{X}_{n}$-scheme. There is a natural morphism of ringed topoi

$$
j: \widehat{\mathcal{X}} \rightarrow \mathcal{X}_{e t} .
$$

If $\mathcal{F}$ is a sheaf of $\mathcal{O}_{\mathcal{X}}$-modules, then we denote by $\widehat{\mathcal{F}}$ the sheaf $j^{*} \mathcal{F}$. Note that the functor $\mathcal{F} \mapsto \widehat{\mathcal{F}}$ is an exact functor.

Lemma 2.2. If $\mathcal{F}$ and $\mathcal{G}$ are coherent sheaves on $\mathcal{X}$ with proper support over $A$, then for every integer $n$ the natural map

$$
\operatorname{Ext}_{\mathcal{O}_{\mathcal{X}}}^{n}(\mathcal{F}, \mathcal{G}) \longrightarrow \operatorname{Ext}_{\mathcal{O}_{\widehat{\mathcal{X}}}}^{n}(\widehat{\mathcal{F}}, \widehat{\mathcal{G}})
$$

is an isomorphism.

Proof. Observe first that the natural map

$$
\mathcal{E} x t_{\mathcal{O}_{\mathcal{X}}}^{n}(\mathcal{F}, \mathcal{G})^{\wedge} \rightarrow \mathcal{E} x t_{\mathcal{O}_{\widehat{\mathcal{X}}}}^{n}(\widehat{\mathcal{F}}, \widehat{\mathcal{G}})
$$

is an isomorphism. Indeed this can be verified locally and so follows from the theory of formal schemes. From this and the local-to-global spectral sequence for Ext it follows that it suffices to show that for any coherent sheaf $\mathcal{F}$ with proper support, the natural map

$$
H^{n}(\mathcal{X}, \mathcal{F}) \longrightarrow H^{n}(\widehat{\mathcal{X}}, \widehat{\mathcal{F}})
$$

is an isomorphism for every $n$. We prove this by induction on the dimension of $\mathcal{X}$.

The key observation is that if $a: U \rightarrow \mathcal{X}$ is a finite morphism from a scheme to $\mathcal{X}$, then the map 3 is known to be an isomorphism for all $n$ in the case when $\mathcal{F}$ is equal to $a_{*} \mathcal{F}^{\prime}$ for some coherent sheaf $\mathcal{F}^{\prime}$ on $U$ (since $a$ is finite and [4. III.5).

If the dimension of $\mathcal{X}$ is zero, then we can by $(9,16.6)$ find a finite étale cover $a: U \rightarrow \mathcal{X}$ of $\mathcal{X}$ by a scheme $U$. If $b: U \times \mathcal{X} U \rightarrow \mathcal{X}$ denotes the canonical map, then the sequences

$$
\begin{aligned}
& \mathcal{F} \rightarrow a_{*} a^{*} \mathcal{F} \rightrightarrows b_{*} b^{*} \mathcal{F} \\
& \widehat{\mathcal{F}} \rightarrow a_{*} a^{*} \widehat{\mathcal{F}} \rightrightarrows b_{*} b^{*} \widehat{\mathcal{F}}
\end{aligned}
$$

are exact so the result is true for $n=0$.

To prove the result for general $n$, assume the result is true for $n-1$ and let $\mathcal{G}=a_{*} a^{*} \mathcal{F} / \mathcal{F}$ so that we have an exact sequence of coherent sheaves

$$
0 \rightarrow \mathcal{F} \rightarrow a_{*} a^{*} \mathcal{F} \rightarrow \mathcal{G} \rightarrow 0 .
$$


Then the commutative diagram

$$
\begin{aligned}
& H^{n-1}\left(\mathcal{X}, a_{*} a^{*} \mathcal{F}\right) \quad \rightarrow \quad H^{n-1}(\mathcal{X}, \mathcal{G}) \quad \rightarrow \quad H^{n}(\mathcal{X}, \mathcal{F}) \quad \rightarrow \quad H^{n}\left(\mathcal{X}, a_{*} a^{*} \mathcal{F}\right) \\
& H^{n-1}\left(\stackrel{\downarrow}{\left.\widehat{\mathcal{X}}, a_{*} a^{*} \widehat{\mathcal{F}}\right)} \rightarrow \begin{array}{c}
\downarrow \\
\downarrow
\end{array} H^{n-1}(\widehat{\mathcal{X}}, \widehat{\mathcal{G}}) \rightarrow H^{n}(\stackrel{\downarrow}{\widehat{\mathcal{X}}}, \widehat{\mathcal{F}}) \rightarrow H^{n}\left(\widehat{\mathcal{X}}, a_{*} a^{*} \widehat{\mathcal{F}}\right)\right.
\end{aligned}
$$

shows that the map $H^{n}(\mathcal{X}, \mathcal{F}) \rightarrow H^{n}(\widehat{\mathcal{X}}, \widehat{\mathcal{F}})$ is injective. But then the map $H^{n}(\mathcal{X}, \mathcal{G}) \rightarrow H^{n}(\widehat{\mathcal{X}}, \widehat{\mathcal{G}})$ is also injective, and an analysis of the diagram

$$
\begin{aligned}
& H^{n-1}(\mathcal{X}, \mathcal{G}) \rightarrow H^{n}(\mathcal{X}, \mathcal{F}) \rightarrow H^{n}\left(\mathcal{X}, a_{*} a^{*} \mathcal{F}\right) \rightarrow H^{n}(\mathcal{X}, \mathcal{G}) \\
& \downarrow \\
& H^{n-1}(\widehat{\mathcal{X}}, \widehat{\mathcal{G}}) \rightarrow H^{n}(\widehat{\mathcal{X}}, \widehat{\mathcal{F}}) \rightarrow H^{n}\left(\widehat{\mathcal{X}}, a_{*} a^{*} \widehat{\mathcal{F}}\right) \rightarrow H^{n}(\widehat{\mathcal{X}}, \widehat{\mathcal{G}})
\end{aligned}
$$

reveals that the map $H^{n}(\mathcal{X}, \mathcal{F}) \rightarrow H^{n}(\widehat{\mathcal{X}}, \widehat{\mathcal{F}})$ is an isomorphism. This completes the proof of the case when $\operatorname{dim}(\mathcal{X})=0$.

To prove the result for general $\mathcal{X}$ we assume the result is true for $\operatorname{dim}(\mathcal{X})-$ 1 and proceed by induction on $n$. By $([9,16.6)$ there exists a finite surjective morphism $a: U \rightarrow \mathcal{X}$ which is generically étale. Let $b: U \times \mathcal{X} U \rightarrow \mathcal{X}$ be the natural map and let $K$ be the kernel of $a_{*} a^{*} \mathcal{F} \rightarrow b_{*} b^{*} \mathcal{F}$. Note that the map 3 for $K$ is an isomorphism when $n=0$. There is a natural map $\mathcal{F} \rightarrow K$ which is generically an isomorphism. Let $\mathcal{F}^{\prime}$ be the kernel of this map and let $\mathcal{F}^{\prime \prime}$ be the cokernel. Then $\mathcal{F}^{\prime}$ and $\mathcal{F}^{\prime \prime}$ have lower-dimensional support and so the map 3 is an isomorphism for these sheaves. If $\mathcal{G}$ denotes the image of $\mathcal{F} \rightarrow K$, then the map $H^{0}(\mathcal{X}, \mathcal{G}) \rightarrow H^{0}(\widehat{\mathcal{X}}, \widehat{\mathcal{G}})$ is an isomorphism by the corresponding result for $K$ and $\mathcal{F}^{\prime \prime}$ and the exact sequence

$$
0 \rightarrow \mathcal{G} \rightarrow K \rightarrow \mathcal{F}^{\prime \prime} \rightarrow 0 \text {. }
$$

Then from the exact sequence

$$
0 \rightarrow \mathcal{F}^{\prime} \rightarrow \mathcal{F} \rightarrow \mathcal{G} \rightarrow 0
$$

we deduce that the map $H^{0}(\mathcal{X}, \mathcal{F}) \rightarrow H^{0}(\widehat{\mathcal{X}}, \widehat{\mathcal{F}})$ is an isomorphism.

To prove the result for $n$ assuming the result for $n-1$, note that a similar argument to the one above shows that if the map $H^{n}(\mathcal{X}, K) \rightarrow H^{n}(\widehat{\mathcal{X}}, \widehat{K})$ is injective (resp. an isomorphism) then the map 3 is injective (resp. an isomorphism). Therefore the proof is completed by using the argument of the case $\operatorname{dim}(\mathcal{X})=0$ with $K$ replacing $\mathcal{F}$.

Now observe that the category of compatible families of coherent sheaves on the $\mathcal{X}_{n}$ with proper support is naturally viewed as a full subcategory of the category of sheaves of $\mathcal{O}_{\widehat{\mathcal{X}}}$-modules on $\widehat{\mathcal{X}}$ using the functor which sends a family $\left\{\mathcal{F}_{n}\right\}$ to the sheaf $\widehat{\mathcal{F}}$ associated to the presheaf

$$
U_{0} \mapsto \lim _{\longleftarrow} \Gamma\left(U_{0}, \mathcal{F}_{n}\right) .
$$

The functor $\left\{\mathcal{F}_{n}\right\} \mapsto \widehat{\mathcal{F}}$ is fully faithful and identifies the category of compatible systems of coherent sheaves on the $\mathcal{X}_{n}$ with proper support with a subcategory of the category of sheaves of $\mathcal{O}_{\hat{\mathcal{X}}}$-modules which is closed under the formation of kernels, cokernels, and extensions. Indeed, these assertions 
can be verified locally on $\mathcal{X}$ and hence follow from the corresponding statements for formal schemes. From this it and the lemma it follows that the functor in 2.1 is fully faithful.

Now the functor from coherent sheaves on $\mathcal{X}$ with proper support to sheaves of $\mathcal{O}_{\widehat{\mathcal{X}}}$-modules on $\widehat{\mathcal{X}}$ identifies the category of such sheaves with a full subcategory of the category of sheaves of $\mathcal{O}_{\widehat{\mathcal{X}}}$-modules which is stable under the formation of kernels, cokernels, and extensions (by lemma 2.2). Thus the following two lemmas prove proposition 2.1.

Lemma 2.3. Let $\mathcal{A}$ be an abelian category and let $\mathcal{A}^{\prime} \subset \mathcal{A}$ be a full subcategory which is stable under the formation of kernels, cokernels, and extensions. Then any object of $\mathcal{A}$ which admits a morphism to an object of $\mathcal{A}^{\prime}$ such that the kernel and cokernel are in $\mathcal{A}^{\prime}$ is in $\mathcal{A}^{\prime}$.

Proof. By assumption such an object $\mathcal{F} \in \mathcal{A}$ sits in an exact sequence

$$
0 \rightarrow K \rightarrow \mathcal{F} \rightarrow \mathcal{F}^{\prime} \rightarrow Q \rightarrow 0
$$

where $K, \mathcal{F}^{\prime}, Q \in \mathcal{A}^{\prime}$. Let $K^{\prime}=\operatorname{Ker}\left(\mathcal{F}^{\prime} \rightarrow Q\right)$. Then $K^{\prime} \in \mathcal{A}^{\prime}$, and we have an exact sequence

$$
0 \rightarrow K \rightarrow \mathcal{F} \rightarrow K^{\prime} \rightarrow 0
$$

Therefore $\mathcal{F} \in \mathcal{A}^{\prime}$.

Lemma 2.4. For every compatible family of coherent sheaves $\left\{\mathcal{F}_{n}\right\}$ with proper support and associated sheaf $\widehat{\mathcal{F}}$ on $\widehat{\mathcal{X}}$, there exists a morphism $\widehat{\mathcal{F}} \rightarrow$ $j^{*} \mathcal{G}$ for some coherent sheaf $\mathcal{G}$ on $\mathcal{X}$ with proper support such that the kernel and cokernel are isomorphic to the pullbacks of coherent sheaves on $\mathcal{X}$ with proper support.

Proof. We proceed by induction on the dimension of the support of $\widehat{\mathcal{F}}$.

If the support of $\widehat{\mathcal{F}}$ has dimension zero, then for any étale cover $a: U \rightarrow \mathcal{X}$ the inverse image of the support of $\widehat{\mathcal{F}}$ in $\widehat{U}$ is a closed subscheme which is of dimension zero, hence is proper. Therefore, there exists a unique sheaf on $U$ inducing the restriction of $\widehat{\mathcal{F}}$ to $\widehat{U}$ (by 4. III.5). Moreover, by the uniqueness this sheaf comes with descent datum relative to $a$. Hence $\widehat{\mathcal{F}}$ is induced from a coherent sheaf on $\mathcal{X}$ with proper support.

As for the general case, choose a morphism $a: U \rightarrow \mathcal{X}$ which is finite and generically étale (such a morphism exists by [9], 16.6), and let $b: U \times \mathcal{X} U \rightarrow$ $\mathcal{X}$ be the natural map. Then $a_{*} a^{*} \widehat{\mathcal{F}}$ and $b_{*} b^{*} \widehat{\mathcal{F}}$ are obtained from coherent sheaves on $\mathcal{X}$, and hence so is

$$
K:=K \operatorname{Ker}\left(a_{*} a^{*} \widehat{\mathcal{F}} \rightarrow b_{*} b^{*} \widehat{\mathcal{F}}\right) .
$$

Moreover, there is a natural map $\widehat{\mathcal{F}} \rightarrow K$ which is generically an isomorphism. Hence the kernels and cokernels have lower dimensional support and by induction are obtained from coherent sheaves on $\mathcal{X}$. 
Separation Conditions. These follow by the same reasoning as in (2], page $64)$.

\section{Deformation theory.}

Suppose given a deformation situation (in the sense of [2])

$$
A^{\prime} \rightarrow A \rightarrow A_{0}
$$

and a quotient $\mathcal{F}_{A} \rightarrow \mathcal{G}_{A}$ over $\mathcal{X}_{A}=\mathcal{X} \times{ }_{S} \operatorname{Spec}(A)$ giving an element of $Q(A)$. Suppose further that a map $B \rightarrow A$ is given. Then it is well-known (see for example [11, 3.4) that the map

$$
Q\left(A^{\prime} \times_{B} A\right) \longrightarrow Q\left(A^{\prime}\right) \times_{Q(A)} Q(B)
$$

is a bijection.

If $M=\operatorname{Ker}\left(A^{\prime} \rightarrow A\right)$, then it follows from the bijectivity of 4 that a deformation theory in the sense of ([2]) is provided by the module $Q_{\mathcal{G}_{0}}\left(A_{0}[M]\right)$ (see for example loc. cit. page 47). Here $A_{0}[M]$ denotes the ring with underlying module $A_{0} \oplus M$ and multiplication

$$
(a, m) \cdot\left(a^{\prime}, m^{\prime}\right)=\left(a a^{\prime}, a m^{\prime}+a^{\prime} m\right),
$$

and $Q_{\mathcal{G}_{0}}\left(A_{0}[M]\right)$ denotes the set of elements in $Q\left(A_{0}[M]\right)$ whose image in $Q\left(A_{0}\right)$ is the reduction $\mathcal{G}_{0}$ of $\mathcal{G}$. The conditions on the deformation theory of ([2, theorem 5.3) are therefore satisfied by the following lemma and standard properties of cohomology.

Lemma 2.5. Let $\mathcal{H}_{0}=\operatorname{Ker}\left(\mathcal{F}_{0} \rightarrow \mathcal{G}_{0}\right)$ (where $\mathcal{F}_{0}$ denotes the reduction of $\mathcal{F}$ to $\left.\mathcal{X}_{A_{0}}\right)$. Then there is a natural $A_{0}$-module isomorphism

$$
Q_{\mathcal{G}_{0}}\left(A_{0}[M]\right) \simeq \operatorname{Ext} t^{0}\left(\mathcal{H}_{0}, \mathcal{G}_{0} \otimes M\right) .
$$

Proof. Let

$$
0 \longrightarrow \mathcal{G}_{0} \otimes M \longrightarrow \mathcal{G}_{0} \otimes M \oplus \mathcal{F}_{0} \longrightarrow \mathcal{F}_{0} \longrightarrow 0
$$

be the sequence obtained by pushing out the sequence

$$
0 \longrightarrow \mathcal{F}_{0} \otimes M \longrightarrow \mathcal{F}_{0} \oplus \mathcal{F}_{0} \otimes M \longrightarrow \mathcal{G}_{0} \longrightarrow 0
$$

via the given map $\mathcal{F}_{0} \rightarrow \mathcal{G}_{0}$. Then to give a lifting of $\mathcal{G}_{0}$ is equivalent to giving a sub- $\mathcal{O}_{\mathcal{X}_{A_{0}}}$-module $\mathcal{H} \subset \mathcal{F}_{0} \oplus \mathcal{G}_{0} \otimes M$ such that the induced map to $\mathcal{F}_{0}$ induces an isomorphism $\mathcal{H} \simeq \mathcal{H}_{0}$. Note that such a sub-module is automatically a sub- $\mathcal{O}_{\mathcal{X}_{A_{0}[M]}}$-module. In other words, the set of liftings of $\mathcal{G}_{0}$ is in natural bijection with the set of maps $\mathcal{H}_{0} \rightarrow \mathcal{F}_{0} \oplus \mathcal{G}_{0} \otimes M$ lifting the inclusion $\mathcal{H}_{0} \subset \mathcal{F}_{0}$. But to give such a map is precisely equivalent to giving a morphism $\mathcal{H}_{0} \rightarrow \mathcal{G}_{0} \otimes M$. The verification that this bijection is a module homomorphism is left to the reader.

Conditions on the obstructions.

Our understanding of the obstruction theory of $Q$ will be in a 2-step approach (correcting a mistake in [2]). Let $A^{\prime} \rightarrow A \rightarrow A_{0}$ be a deformation 
situation as above and $\mathcal{F}_{A} \rightarrow \mathcal{G}_{A}$ an object in $Q(A)$. For any quotient $\epsilon: M \rightarrow M_{\epsilon}$, let $A_{\epsilon}$ be the quotient of $A^{\prime}$ by the kernel of $\epsilon$. For such an $\epsilon$, the first obstruction to lifting $\mathcal{G}_{A}$ to $A_{\epsilon}$ is that the map

$$
M \otimes \mathcal{F}_{A_{0}} \longrightarrow M_{\epsilon} \otimes \mathcal{G}_{A_{0}}
$$

factors through $M \mathcal{F}_{A^{\prime}}$. If we let $\mathcal{T}$ be the kernel of $M \otimes \mathcal{F}_{A_{0}} \rightarrow \mathcal{F}_{A^{\prime}}$, then we want that the map

$$
\mathcal{T} \longrightarrow M_{\epsilon} \otimes \mathcal{G}_{A_{0}}
$$

is zero. If this is the case, then there is a canonical map $M \mathcal{F}_{A^{\prime}} \rightarrow M_{\epsilon} \otimes$ $\mathcal{G}_{A_{0}}$ and the condition that there exists an element in $Q\left(A_{\epsilon}\right)$ inducing $\mathcal{G}$ is equivalent to the statement that the resulting extension

$$
0 \rightarrow M_{\epsilon} \otimes \mathcal{G}_{A_{0}} \rightarrow E \rightarrow \mathcal{F}_{A} \rightarrow 0
$$

is obtained from an extension of $\mathcal{G}_{A}$ by $M_{\epsilon} \otimes \mathcal{G}_{A_{0}}$. In other words, if the map 5 is zero, then if we let $\mathcal{H}=\operatorname{Ker}\left(\mathcal{F}_{A} \rightarrow \mathcal{G}_{A}\right)$ there exists a canonical obstruction in

$$
\operatorname{Ext}_{\mathcal{X}_{A^{\prime}}}^{1}\left(\mathcal{H}, M_{\epsilon} \otimes \mathcal{G}_{A_{0}}\right)
$$

whose vanishing is necessary and sufficient for the existence of a lifting.

Now the only condition on the obstructions in $(2,5.3)$ which does not follow immediately from the bijectivity of 4 is condition $\left(5.3,\left[5^{\prime}\right] . c\right)$.

Thus suppose given a deformation situation as before, $\xi \in Q(A)$, and suppose further that $M$ is free of rank $n$. Let $K$ be the field of fractions of $A_{0}$, and denote by a subscript $K$ the localizations at the generic point of $\operatorname{Spec}\left(A_{0}\right)$. We suppose that for every one-dimensional quotient $M_{K} \rightarrow M_{K}^{*}$ there is a non-trivial obstruction to lifting $\xi_{K}$ to $Q\left(A_{K}^{*}\right)$, where $A_{K}^{*}$ denotes the extension defined by $M_{K}^{*}$. Then we have to show that there exists a non-empty open subset $U \subset \operatorname{Spec}\left(A_{0}\right)$ such that for every quotient $\epsilon$ of $M$ of length one with support in $U, \xi$ does not lift to $A_{\epsilon}$ (the extension obtained from $\epsilon$ ).

Let

$$
\phi \in \operatorname{Ext}^{0}\left(\mathcal{T}, M \otimes \mathcal{G}_{A_{0}}\right) \simeq \operatorname{Ext}^{0}\left(\mathcal{T}, \mathcal{G}_{A_{0}}\right) \otimes M
$$

be the class defined by 5 in the case when $\epsilon$ is the identity. We reduce to the case when $\phi=0$. Once this reduction is made the argument of ([2], page 66) will finish the proof.

To make the reduction, we can by shrinking on $\operatorname{Spec}\left(A_{0}\right)$ assume that

$$
\operatorname{Ext}^{0}\left(\mathcal{T}, \mathcal{G}_{A_{0}}\right)
$$

is a free module; say of rank $r$. In addition, by the argument of ([2], page 66), we can after shrinking $\operatorname{Spec}\left(A_{0}\right)$ assume that for each point $s \in \operatorname{Spec}\left(A_{0}\right)$, the natural map

$$
\operatorname{Ext}^{0}\left(\mathcal{T}, \mathcal{G}_{A_{0}}\right) \otimes k(s) \longrightarrow \operatorname{Ext}^{0}\left(\mathcal{T}, \mathcal{G}_{A_{0}} \otimes k(s)\right)
$$

is an isomorphism. 
Choosing a basis for $\operatorname{Ext}^{0}\left(\mathcal{T}, \mathcal{G}_{A_{0}}\right)$ we can think of $\phi$ as an element

$$
\phi=\left(\phi_{1}, \ldots, \phi_{r}\right) \in M^{r} .
$$

Let $N \subset M$ be the submodule of $M$ generated by the $\phi_{i}$, and let $M^{\prime}=M / N$. After further shrinking $\operatorname{Spec}\left(A_{0}\right)$ we can assume that $M^{\prime}$ is a free module. Now note that any length-one quotient $M \rightarrow M_{\epsilon}$ for which the obstruction to lifting $\xi$ goes to zero factors through $M^{\prime}$ by 6 . Moreover, any such quotient which does not factor through $M^{\prime}$ is obstructed. Therefore, we may replace $M$ by $M^{\prime}$ and hence are reduced to the case when $\phi=0$.

Valuative criteria for properness when $\mathcal{F}$ has proper support

Let $R$ be a discrete valuation ring with field of fractions $K$, and let $i$ : $\mathcal{X}_{K} \hookrightarrow \mathcal{X}$ be the inclusion of the generic fiber. We suppose that we have a flat quotient $\mathcal{F}_{K} \rightarrow \mathcal{G}_{K}$ over the generic fiber which we wish to extend to $\mathcal{X}$. For this we take $\mathcal{G}$ to be the image of the map $\mathcal{F} \rightarrow i_{*} \mathcal{G}_{K}$. The image is evidently a coherent sheaf, and has proper support since $\mathcal{F}$ has proper support. It is flat because it is a subsheaf of a torsion free sheaf, and by definition $\mathcal{G}$ induces $\mathcal{G}_{K}$ on the generic fiber.

This completes the proof of theorem 1.1 .

\section{Flattening stratifications}

As a corollary of the representability result in the last section, when the locally finitely-presented sheaf $\mathcal{F}$ has proper support over $S$, we can construct the flattening stratification of $\mathcal{F}$ as an algebraic space. But in fact this algebraic space is representable and quasi-affine over $S$. This is a crucial step in the proof of theorem 1.5. Therefore we include a proof of the following fact:

Proposition 3.1. Suppose $f: Y \rightarrow X$ is a finite-type, separated, quasifinite morphism of algebraic spaces. Then $f$ is quasi-affine, in particular $f$ is representable by schemes.

Proof. We need to prove that the natural morphism of $X$-schemes,

$$
\iota: Y \rightarrow \operatorname{Spec}_{X} f_{*} \mathcal{O}_{Y}
$$

is an open immersion. By [8, proposition II.4.18], $f_{*} \mathcal{O}_{Y}$ commutes with flat base change on $X$, so the formation of $\iota$ commutes with flat base change on $X$. Moreover one may check that a morphism of $X$-schemes is an open immersion after fpqc base change. Thus, without loss of generality, we may suppose that $X$ is an affine scheme.

To prove $\iota$ is an open immersion, it suffices to check that for each point $p \in Y$, the following two conditions hold:

(1) $\iota$ is étale at $p$, and 
(2) there is an open set $p \in U \subset Y$ which is disjoint from the image of $Y \times_{\iota, \iota} Y-\Delta(Y)$ under the projection $\operatorname{pr}_{1}: Y \times_{\iota, \iota} Y \rightarrow Y$.

Here $Y \times_{\iota, \iota} Y$ is the fiber product of $Y$ with itself over Spec ${ }_{X} f_{*} \mathcal{O}_{Y}$ and $\Delta: Y \rightarrow Y \times_{\iota, \iota} Y$ is the diagonal morphism. It is clear that if both (1) and (2) hold for each point $p \in Y$, then $\iota$ is an étale monomorphism, and therefore $\iota$ is an open immersion.

The claim is that for given $p \in Y$, we may check (1) and (2) after passing to an étale neighborhood of $q=f(p) \in X$, i.e. if $X^{\prime} \rightarrow X$ is an étale morphism, $q^{\prime} \in X^{\prime}$ is a point lying over $q$, and if $p^{\prime} \in Y^{\prime}:=X^{\prime} \times_{X} Y$ is the point lying over $q^{\prime}$ and $p$, then it suffices to check (1) and (2) for $p^{\prime}$. We have mentioned that the natural morphism of $X^{\prime}$-schemes

$$
\iota^{\prime}: Y^{\prime} \rightarrow \operatorname{Spec}_{X^{\prime}}\left(f^{\prime}\right)_{*} \mathcal{O}_{Y^{\prime}}
$$

is the base-change of $\iota$. The property of being étale at a point can be checked after étale (and even flat) base-change, so if (1) holds for $p^{\prime}$ then (1) holds for $p$. Suppose (2) holds for $p^{\prime}$ and let $U^{\prime}$ be an open set $p^{\prime} \in U^{\prime} \subset Y^{\prime}$ as in (2). Let $p \in U \subset Y$ be the open image of $U^{\prime}$ under $g: Y^{\prime} \rightarrow Y$. We have the equality

$$
\begin{array}{r}
g\left(U^{\prime}\right) \cap \operatorname{pr}_{1}\left(Y \times_{\iota, \iota} Y-\Delta(Y)\right)=g\left(U^{\prime} \cap g^{-1} \operatorname{pr}_{1}\left(Y \times_{\iota, \iota} Y-\Delta(Y)\right)\right)= \\
g\left(U^{\prime} \cap \operatorname{pr}_{1}^{\prime}\left(Y^{\prime} \times_{\iota^{\prime}, \iota^{\prime}} Y^{\prime}-\Delta^{\prime}\left(Y^{\prime}\right)\right)\right) .
\end{array}
$$

But $U^{\prime} \cap \operatorname{pr}_{1}^{\prime}\left(Y^{\prime} \times \times_{\iota^{\prime}, \iota^{\prime}} Y^{\prime}-\Delta^{\prime}\left(Y^{\prime}\right)\right)=\emptyset$ by assumption. Thus $U=g\left(U^{\prime}\right)$ satisfies condition (2) for $p \in Y$. So it suffices to check (1) and (2) for $p^{\prime} \in Y^{\prime}$.

Now let $Z \rightarrow Y$ be an étale morphism of a scheme $Z$ to $Y$ and $r \in Z$ a point mapping to $p$. Then $Z \rightarrow X$ is finite-type, separated and quasifinite. By [3, proposition 2.3.8(a)], we may find an étale morphism $X^{\prime} \rightarrow X$ with $X^{\prime}$ an affine scheme, and a point $q^{\prime} \in X^{\prime}$ mapping to $q=f(p)$ such that if $r^{\prime} \in Z^{\prime}$ is the point lying over $q^{\prime}$ and $r$, and if $Z_{0}^{\prime}$ is the connected component of $Z^{\prime}$ containing $r^{\prime}$, then $Z_{0}^{\prime} \rightarrow X^{\prime}$ is a finite morphism of affine schemes. Let $Y_{0}^{\prime} \subset Y^{\prime}$ be the connected component of $Y^{\prime}$ containing the image of $Z_{0}^{\prime}$. Then $Z_{0}^{\prime} \rightarrow Y_{0}^{\prime}$ is étale with dense image, but it is also proper. Therefore $Z_{0}^{\prime} \rightarrow Y_{0}^{\prime}$ is finite,étale and surjective. So, by Knudsen's version of Chevalley's theorem [8, theorem III.4.1], $Y_{0}^{\prime}$ is an affine scheme.

Let $Y_{r}^{\prime}$ denote the union of connected components $Y^{\prime}-Y_{0}^{\prime}$. Then we have a decomposition of $\operatorname{Spec}_{X^{\prime}}\left(f^{\prime}\right)_{*} \mathcal{O}_{Y^{\prime}}$ into components, $\operatorname{Spec}_{X^{\prime}}\left(f_{0}^{\prime}\right)_{*} \mathcal{O}_{Y_{0}^{\prime}}$ union $\operatorname{Spec}_{X^{\prime}}\left(f_{r}^{\prime}\right)_{*} \mathcal{O}_{Y_{r}^{\prime}}$ and a decomposition of $\iota^{\prime}$ as the "disjoint union" of the two morphisms:

$$
\begin{aligned}
& \iota_{0}^{\prime}: Y_{0}^{\prime} \rightarrow \operatorname{Spec}_{X^{\prime}}\left(f_{0}^{\prime}\right)_{*} \mathcal{O}_{Y_{0}^{\prime}}, \\
& \iota_{r}^{\prime}: Y_{r}^{\prime} \rightarrow \operatorname{Spec}_{X^{\prime}}\left(f_{r}^{\prime}\right)_{*} \mathcal{O}_{Y_{r}^{\prime}} .
\end{aligned}
$$


And $\iota^{\prime}$ is an isomorphism. Thus $\iota^{\prime}$ is étale at $p^{\prime}$. And defining $U^{\prime}=Y_{0}^{\prime}$, we see that $U^{\prime}$ satisfies the condition (2) for $p^{\prime} \in Y^{\prime}$. So (1) and (2) hold for $p^{\prime}$ and thus (1) and (2) hold for $p \in Y$. Since this holds for every $p \in Y$, we conclude that $\iota$ is an open immersion, i.e. $f: Y \rightarrow X$ is quasi-affine.

Remark: We could not find precisely this statement in [8], although it follows easily from results proved there. If one further assumes that $f$ is finitely-presented, then this result also follows easily from [9, théorème 16.5].

Now suppose $S$ is an algebraic space, $f: \mathcal{X} \rightarrow S$ is a 1-morphism from a Deligne-Mumford stack to $S$ which is separated and locally finitelypresented. Let $\mathcal{F}$ be a locally finitely-presented $\mathcal{O}_{\mathcal{X}}$-module. Consider the functor

$$
\Sigma:(S-\text { schemes })^{\mathrm{opp}} \rightarrow \text { Sets, }
$$

which to any morphism $g: T \rightarrow S$ associates $\{*\}$ if the pullback of $\mathcal{F}$ to $T \times_{S} \mathcal{X}$ is flat over $T$, and which associates $\emptyset$ otherwise. Given a morphism of $S$-schemes, $h: T_{1} \rightarrow T_{2}$, the morphism $\Sigma(h)$ is defined to be the unique map $\Sigma\left(T_{2}\right) \rightarrow \Sigma\left(T_{1}\right)$. For this to make sense, we must check that if $\Sigma\left(T_{2}\right)$ is nonempty, then so is $\Sigma\left(T_{1}\right)$. But this is clear, if the pullback of $\mathcal{F}$ to $T_{2} \times{ }_{S} \mathcal{X}$ is flat over $T_{2}$, then the pullback of this sheaf over $T_{1} \times_{T_{2}}\left(T_{2} \times_{S} \mathcal{X}\right)$ is flat over $T_{1}$. And this pullback is isomorphic to the pullback of $\mathcal{F}$ to $T_{1} \times{ }_{S} \mathcal{X}$. So $\Sigma\left(T_{1}\right)$ is nonempty.

Theorem 3.2. Let $f: \mathcal{X} \rightarrow S, \mathcal{F}$, and $\Sigma$ be as above.

(1) $\Sigma$ is an fpqc sheaf which is limit preserving and $\Sigma \rightarrow S$ is a monomorphism.

(2) If $\mathcal{F}$ has proper support over $S$, then $\Sigma$ is an algebraic space and $\Sigma \rightarrow$ $S$ is a surjective, finitely-presented monomorphism. In particular, $\Sigma \rightarrow S$ is quasi-affine.

Proof. It is immediate that $\Sigma \rightarrow S$ is a monomorphism. Since one may check that a quasi-coherent sheaf on $T \times_{S} \mathcal{X}$ is flat over $T$ after performing an fpqc base change of $T$, it follows that $\Sigma$ is an fpqc sheaf. The fact that $\Sigma$ is limit-preserving follows from [4, théorème IV.11.2.6]. So (1) is proved.

To prove (2), first notice that we may use (1) to reduce to the case that $S=$ Spec $A$ is a Noetherian affine scheme, and $\mathcal{X}$ is the support of $\mathcal{F}$ which is a proper, finitely-presented Deligne-Mumford stack over $S$. By theorem 1.1 we know the Quot functor of $\mathcal{F}$ is represented by an algebraic space $Q \rightarrow S$ which is separated and locally finitely-presented. Denote by

$$
0 \longrightarrow \mathcal{K} \longrightarrow \operatorname{pr}_{2}^{*} \mathcal{F} \longrightarrow \mathcal{G} \longrightarrow 0
$$

the universal short exact sequence on $Q \times{ }_{S} \mathcal{X}$. Since $\operatorname{pr}_{2}^{*} \mathcal{F}$ has proper support over $Q, \mathcal{K}$ also has proper support over $Q$. Define $U$ to be the complement in $Q$ of the image of the support of $\mathcal{K}$. The restriction of the universal short 
exact sequence over $U$ is simply the identity morphism of $\operatorname{pr}_{2}^{*} \mathcal{F}$ to itself, i.e. the pullback of $\mathcal{F}$ to $U$ is flat over $U$. So we have an induced morphism $U \rightarrow \Sigma$. Conversely there is an obvious morphism $\Sigma \rightarrow Q$ which factors through $U$, and we see that $U \rightarrow \Sigma$ is a natural isomorphism of algebraic spaces. So $\Sigma$ is an algebraic space and $\Sigma \rightarrow S$ is a locally finitely-presented monomorphism.

Any module over a field is flat over that field, therefore for each field $K$ and each morphism Spec $K \rightarrow S$, this morphism factors through $\Sigma \rightarrow S$. So $\Sigma \rightarrow S$ is surjective. The claim is that for any surjective, locally finitelypresented monomorphism $h: Y \rightarrow S$ of an algebraic space to a Noetherian affine scheme, $Y$ is quasi-compact.

We will prove this claim by induction on the dimension of $S$. If $S=$ Spec $K$ for some field $K$, it is obvious. If $Y^{\text {red }}$ is quasi-compact, the same is true of $Y$, so we may reduce to the case that $S$ is reduced. A finite union of quasi-compact sets is quasi-compact, so we may reduce to the case that $S$ is integral. Now suppose the result is proved for all schemes $S$ of dimension at most $n$ and suppose $S$ is an integral scheme of dimension $n+1$. Let Spec $K$ be the generic point of $S$ and let $U \subset Y$ be the open set where $U \rightarrow S$ is étale. Then Spec $K$ factors through $Y$, and in fact it is contained in $U$. Thus $U \rightarrow S$ is an étale monomorphism, i.e. an open immersion which has dense image. Let the complement of $U$ in $S$ be $C$ (with reduced scheme structure) and let the preimage of $C$ be $Z$. Then $Z \rightarrow C$ is again a surjective, locally finitely-presented monomorphism, and $C$ has dimension at most $n$. So by the induction assumption, $Z$ is quasi-compact. Since $U$ is an open subset of a Noetherian scheme, it is quasi-compact. Thus $Y=U \cup Z$ is quasi-compact and the claim is proved by induction.

By the last paragraph, we conclude that $\Sigma \rightarrow S$ is a surjective, finitelypresented monomorphism of algebraic spaces. So by proposition 3.1, we conclude that $\Sigma \rightarrow S$ is quasi-affine.

Remarks: (1) If the support of $\mathcal{F}$ is a scheme and the morphism to $S$ is projective, then it follows from [10, theorem, p.55] that $\Sigma \rightarrow S$ is a disjoint union of locally closed immersions. While one can find examples of surjective, finitely-presented monomorphisms $Y \rightarrow S$ not a disjoint union of locally closed immersions, we conjecture that $\Sigma \rightarrow S$ is a disjoint union of locally closed immersions whenever $\mathcal{F}$ has proper support over $S$.

(2) Again in the case that the support of $\mathcal{F}$ is projective over $S$, the methods in [10, section 8] provide a global construction of the flattening stratification $\Sigma \rightarrow S$ along with a partition labelled by the Hilbert polynomial of the fibers of $\mathcal{F}$. In the case that $\mathcal{X}$ is a tame, global quotient 
with projective coarse moduli space, we believe that there is again a global construction of the flattening stratification $\Sigma \rightarrow S$ along with a partition labelled by the Hilbert polynomial (as defined in section 4). However we don't know what this global construction is, and the proof of the existence of $\Sigma \rightarrow S$ given above is the one truly non-constructive step in the proof of theorm 1.5

\section{Hilbert POLYNOMIAlS}

Recall that a Deligne-Mumford stack $\mathcal{X}$ is tame if for each algebraicallyclosed field $k$ and each 1-morphism $\zeta$ : Spec $k \rightarrow \mathcal{X}$, the $k$-valued points of

$$
G_{\zeta}:=\operatorname{Spec} k \times_{(\zeta, \zeta), \mathcal{X} \times \mathcal{X}, \Delta} \mathcal{X}
$$

form a group of order prime to $\operatorname{char}(k)$. We remind the reader of some facts about tame Deligne-Mumford stacks.

Lemma 4.1. Let $\mathcal{X}$ be a tame Deligne-Mumford stack, $\pi: \mathcal{X} \rightarrow X$ its coarse moduli space.

(1) The additive functor $\pi_{*}$ from the category of $\mathcal{O}_{\mathcal{X}}$-modules to the category of $\mathcal{O}_{X}$-modules maps quasi-coherent sheaves to quasi-coherent sheaves and maps locally finitely-presented sheaves to locally finitelypresented sheaves.

(2) The additive functor $\pi_{*}$ is exact, in particular $R^{i} \pi_{*} \mathcal{F}=0$ for $i>0$ and $\mathcal{F}$ any quasi-coherent $\mathcal{O}_{\mathcal{X}}$-module.

(3) Suppose $g: X \rightarrow S$ is a morphism of algebraic spaces and suppose $\mathcal{F}$ is a quasi-coherent sheaf on $\mathcal{X}$ which is flat over $S$. Then also $\pi_{*} \mathcal{F}$ is flat over $S$.

Proof. (1) and (2) form [1, lemma 2.3.4]. And (3) follows by the same local analysis in the proof of [1, lemma 2.3.4] since the invariant submodule $M^{\Gamma}$ (in the notation of [1]) of an $S$-flat module $M$ is a direct summand, and so it is also $S$-flat.

Suppose $k$ is a field and $\mathcal{X}$ is a separated, locally finitely-presented tame Deligne-Mumford stack over Spec $k$, and let $\pi: \mathcal{X} \rightarrow X$ be its coarse moduli space. Suppose that $\mathcal{F}$ is a coherent $\mathcal{O}_{\mathcal{X}}$-module with proper support. Then by lemma 4.1, $H_{\text {ét }}^{i}(\mathcal{X}, \mathcal{F})=H_{\text {ét }}^{i}\left(X, \pi_{*} \mathcal{F}\right)$ for all $i$, and hence these groups are zero for $i$ bigger than the dimension of $\mathcal{X}$. Therefore the sum

$$
\chi(\mathcal{X}, \mathcal{F})=\sum_{i=0}^{\infty}(-1)^{i} \operatorname{dim}_{k} H_{\text {ét }}^{i}(\mathcal{X}, \mathcal{F})
$$

is finite. For each locally free sheaf $E$ of finite rank on $\mathcal{X}$, we also have that $E \otimes_{\mathcal{O}_{\mathcal{X}}} \mathcal{F}$ is again coherent with proper support. Since $E \mapsto \chi\left(\mathcal{X}, E \otimes_{\mathcal{O}_{\mathcal{X}}} \mathcal{F}\right)$ 
is additive in short exact sequences, we have a well-defined group homomorphism

$$
P_{\mathcal{F}}: K^{0}(\mathcal{X}) \rightarrow \mathbb{Z},[E] \mapsto \chi\left(\mathcal{X}, E \otimes_{\mathcal{O}_{\mathcal{X}}} \mathcal{F}\right)
$$

Definition 4.2. Given a homomorphism of Abelian groups $i: A \rightarrow K^{0}(\mathcal{X})$, define the A-Hilbert polynomial of $\mathcal{F}, P_{A, \mathcal{F}}$, to be the function $P_{A, \mathcal{F}}=P_{\mathcal{F}} \circ i$.

Remarks: (1) If $\mathcal{L}$ is an invertible sheaf on $\mathcal{X}, A=\mathbb{Z}\left[x, x^{-1}\right]$ and $i$ : $A \rightarrow K^{0}(\mathcal{X})$ is the group homomorphism such that $i\left(x^{d}\right)=\left[\mathcal{L}^{d}\right]$, then the $A$-Hilbert polynomial of $\mathcal{F}, P_{A, \mathcal{F}}$ is the usual Hilbert polynomial of $\mathcal{F}$ with respect to $\mathcal{L}$. We will need to consider cases where $i: A \rightarrow K^{0}(\mathcal{F})$ cannot be reduced to this form, which is why our notion of Hilbert polynomial is so general.

(2) The most instructive example, from our point of view, is when $\mathcal{X}=$ $B G$ for some finite, étale $k$-group scheme $G$. Then $K^{0}(B G)$ is naturally isomorphic to the Grothendieck group of the category of finite $k[G]$-modules, i.e. the representation ring of $k[G]$. And the Hilbert polynomial $P_{\mathcal{F}}$ determines the image $[\mathcal{F}]$ of $\mathcal{F}$ in $K^{0}(B G)$.

Now suppose that $f: \mathcal{X} \rightarrow S$ is a 1-morphism from a tame DeligneMumford stack to a connected algebraic space such that $f$ is separated and locally finitely-presented, Define $A=K^{0}(\mathcal{X})$ and for each field $k$ and each morphism $g$ : Spec $k \rightarrow S$, define $i_{g}: A \rightarrow K^{0}\left(\operatorname{Spec} k \times_{S} \mathcal{X}\right)$ to be the pullback map $K^{0}\left(\mathrm{pr}_{2}\right)$.

Lemma 4.3. Suppose that $\mathcal{F}$ is a locally finitely-presented quasi-coherent sheaf on $\mathcal{X}$ which is flat over $S$ and which has proper support over $S$. Then there exists a function $P: A \rightarrow \mathbb{Z}$ such that for all $g:$ Spec $k \rightarrow S, P_{A, g^{*} \mathcal{F}}=$ $P$.

Proof. We need to show that for each locally free sheaf $\mathcal{E}$ on $\mathcal{X}$, the function

$$
(g: \operatorname{Spec} k \rightarrow S) \mapsto \chi\left(g^{*}\left(\mathcal{E} \otimes_{\mathcal{O}_{\mathcal{X}}} \mathcal{F}\right)\right)
$$

is constant. Since $R^{i} \pi_{*}$ vanishes on all quasi-coherent modules for $i>0$, we have

$$
\chi\left(g^{*}\left(\mathcal{E} \otimes_{\mathcal{O}_{\mathcal{X}}} \mathcal{F}\right)\right)=\chi\left(g^{*} \pi_{*}\left(\mathcal{E} \otimes_{\mathcal{O}_{\mathcal{X}}} \mathcal{F}\right)\right) .
$$

And by lemma $4.1(3)$, we know that $\pi_{*}\left(\mathcal{E} \otimes_{\mathcal{O}_{\mathcal{X}}} \mathcal{F}\right)$ is an $S$-flat, locally finitelypresented sheaf with proper support over $S$. So by [10, corollary, p. 50], we conclude that there is some $P([\mathcal{E}]) \in \mathbb{Z}$ such that for all $g:$ Spec $k \rightarrow S$, we have

$$
\chi\left(g^{*} \pi_{*}\left(\mathcal{E} \otimes_{\mathcal{O}_{\mathcal{X}}} \mathcal{F}\right)\right)=P([\mathcal{E}]) .
$$


Fix an additive homomorphism $P: K^{0}(\mathcal{X}) \rightarrow \mathbb{Z}$ and define $Q^{P}=$ $Q^{P}(\mathcal{F} / \mathcal{X} / S)$ to be the subfunctor of $Q(\mathcal{F} / \mathcal{X} / S)$ such that for each $S$ scheme, $g: Z \rightarrow S$ we have $Q^{P}(g: Z \rightarrow S)$ is the set of quotients $\left[g^{*} \mathcal{F} \rightarrow \mathcal{G}\right] \in Q(g: Z \rightarrow S]$ such that for each field $k$ and each morphism $h:$ Spec $k \rightarrow Z$, we have $P_{A, h^{*} \mathcal{G}}=P$. By lemma 4.3, we see that $Q^{P}$ is an open and closed subfunctor (possibly empty) of $Q$ and that $Q$ is the disjoint union of $Q^{P}$ as $P$ ranges over all $P$.

Observe that theorem 1.5 is implied by the following refinement.

Theorem 4.4. Suppose that $S$ is an affine scheme. Suppose that $\mathcal{X}$ is a tame Deligne-Mumford stack which is separated and finitely-presented over $S$, whose coarse moduli space is a quasi-projective $S$-scheme (resp. projective $S$-scheme), and which is a global quotient over $S$. Then for each locally finitely-presented quasi-coherent sheaf $\mathcal{F}$ on $\mathcal{X}$ and for each homomorphism $P: K^{0}(\mathcal{X}) \rightarrow \mathbb{Z}$, the functor $Q^{P}(\mathcal{F} / \mathcal{X} / S)$ is represented by an algebraic space $Q^{P}$ which admits a factorization $Q^{P} \rightarrow Q^{\prime} \rightarrow S$ where $Q^{\prime}$ is projective

over $S$ and $Q^{P} \rightarrow Q^{\prime}$ is a finitely-presented, quasi-finite monomorphism. If $\mathcal{F}$ has proper support over $S$, then $Q^{P} \rightarrow Q^{\prime}$ is a finitely-presented closed immersion.

Remark: In particular, if $S$ is affine, then $Q^{P}(\mathcal{F} / \mathcal{X} / S)$ is represented by a quasi-projective $S$-scheme (which is projective if the support of $\mathcal{F}$ is proper over $S$ ).

We will prove theorem 4.4 in sections 5 and 6 .

\section{Generating sheaves}

Let $\mathcal{X}$ be a tame Deligne-Mumford stack with coarse moduli space $\pi$ : $\mathcal{X} \rightarrow X$. For each locally free sheaf $\mathcal{E}$ on $\mathcal{X}$, define additive functors

$$
\begin{gathered}
F_{\mathcal{E}}: \text { Quasi-coherent }_{\mathcal{X}} \rightarrow \text { Quasi-coherent }_{X}, \\
G_{\mathcal{E}}: \text { Quasi-coherent }_{\mathcal{X}} \rightarrow \text { Quasi-coherent }_{\mathcal{X}}
\end{gathered}
$$

by the formulas

$$
F_{\mathcal{E}}(\mathcal{F})=\pi_{*} \operatorname{Hom}_{\mathcal{O}_{\mathcal{X}}}(\mathcal{E}, \mathcal{F}), \quad G_{\mathcal{E}}=\pi^{*}\left(F_{\mathcal{E}}(\mathcal{F})\right) \otimes_{\mathcal{O}_{\mathcal{X}}} \mathcal{E}
$$

where $\mathcal{F}$ is a quasi-coherent sheaf on $\mathcal{X}$.

By lemma 4.1, $F_{\mathcal{E}}$ is an exact functor which preserves flatness and preserves the property of being locally finitely-presented. And $G_{\mathcal{E}}$ is a rightexact functor which preserves the property of being locally finitely-presented. Moreover there is a natural transformation $\theta_{\mathcal{E}}: G_{\mathcal{E}} \Rightarrow \mathrm{Id}$ where Id is the identity functor on the category of quasi-coherent sheaves on $\mathcal{X}$ and for a 
quasi-coherent sheaf $\mathcal{F}$ on $\mathcal{X}$, the morphism

$$
\theta_{\mathcal{E}}(\mathcal{F}): \pi^{*}\left(\pi_{*} \operatorname{Hom}_{\mathcal{O}_{\mathcal{X}}}(\mathcal{E}, \mathcal{F})\right) \otimes_{\mathcal{O}_{\mathcal{X}}} \mathcal{E} \rightarrow \mathcal{F}
$$

is the left adjoint to the natural morphism

$$
\pi^{*} \pi_{*} \operatorname{Hom}_{\mathcal{O}_{\mathcal{X}}}(\mathcal{E}, \mathcal{F}) \rightarrow \operatorname{Hom}_{\mathcal{O}_{\mathcal{X}}}(\mathcal{E}, \mathcal{F})
$$

which is itself the left adjoint of the identity morphism

$$
\pi_{*} \operatorname{Hom}_{\mathcal{O}_{\mathcal{X}}}(\mathcal{E}, \mathcal{F}) \rightarrow \pi_{*} \operatorname{Hom}_{\mathcal{O}}(\mathcal{E}, \mathcal{F}) .
$$

Remark: Since $\mathcal{X}$ is a tame stack, $\pi_{*}$ from quasi-coherent sheaves on $\mathcal{X}$ to quasi-coherent sheaves on $X$ is compatible with arbitrary base changes $T \rightarrow X$ (see for example the proof of [1, 2.3.3]). It follows that also the functors $F, G$ and the natural transformation $\theta$ are compatible with arbitrary base changes $T \rightarrow X$.

Definition 5.1. With notation as above, $\mathcal{E}$ is a generator for $\mathcal{F}$ if $\theta_{\mathcal{E}}(\mathcal{F})$ is surjective.

Example: Suppose that $G$ is a finite group and $\mathcal{X}=B G \times \times_{\text {Spec } \mathbb{Z}}$ Spec $k$. Then the quasi-coherent $\mathcal{O}_{\mathcal{X}}$-modules correspond to (left-)modules over $k[G]$. Let $\mathcal{E}$ be the locally free sheaf corresponding to the left regular representation of $G$ on $k[G]$. Then for every quasi-coherent sheaf $\mathcal{F}, \mathcal{E}$ is a generator for $\mathcal{F}$. For that matter, if $M$ is any $k[G]$-module which contains every irreducible representation of $G$ as a submodule, then the locally free sheaf corresponding to $M$ is a generator for every $\mathcal{F}$.

The goal of this section is to prove that the previous example is typical for separated, tame, Deligne-Mumford stacks which are global quotients. As suggested by the referee, we first prove the following pointwise condition for a locally free sheaf $\mathcal{E}$ to be a generator.

Theorem 5.2. Suppose that $\mathcal{X}$ is a tame Deligne-Mumford stack as above and $\mathcal{E}$ is a locally free sheaf on $\mathcal{X}$. For each algebraically-closed field $k$ and each 1-morphism $\zeta:$ Spec $k \rightarrow \mathcal{X}$ with stabilizer group $G_{k}$ (c.f. section 4 ), let $\widetilde{\zeta}: B G_{\zeta} \rightarrow \mathcal{X}$ be the natural map.

Suppose that for some 1-morphism $\zeta:$ Spec $k \rightarrow \mathcal{X}$, the locally free sheaf $\mathcal{E}_{\zeta}:=(\widetilde{\zeta})^{*} \mathcal{E}$ is a generator for the left regular representation of $G_{\zeta}$, i.e. considered as a finite-dimensional $k\left[G_{\zeta}\right]$-module, $\mathcal{E}_{\zeta}$ contains every irreducible representation of $G_{\zeta}$ as a submodule. Then there exists a Zariski open subset $U \subset X$ containing the image of $\zeta($ Spec $k)$ such that the restriction of $\mathcal{E}$ to $\mathcal{X} \times_{X} U$ is a generator for every quasi-coherent sheaf on $\mathcal{X} \times_{X} U$. In particular, if $(\widetilde{\zeta})^{*} \mathcal{E}$ is a generator for the left regular representation of $G_{\zeta}$ for every geometric point $\zeta:$ Spec $k \rightarrow \mathcal{X}$, then $\mathcal{E}$ is a generator for every quasi-coherent sheaf on $\mathcal{X}$.

Remarks: (1) The converse to theorem 5.2 is obvious. Suppose that $\mathcal{E}$ is a locally free sheaf on $\mathcal{X}$ and suppose that $\widetilde{\zeta}: B G_{\zeta} \rightarrow \mathcal{X}$ is as above. Let 
$\mathcal{F}$ be the push-forward by $\widetilde{\zeta}$ of the sheaf on $B G_{\zeta}$ corresponding to the left regular representation of $G_{\zeta}$. Then $\mathcal{E}$ is a generator for $\mathcal{F}$ iff $(\widetilde{\zeta})^{*} \mathcal{E}$ is a generator for the left regular representation of $G_{\zeta}$.

(2) Suppose $\mathcal{X}$ is a quotient stack of the form $\mathcal{X}=\left[Y / G L_{n}\right]$. Then for each geometric point $\zeta$ : Spec $k \rightarrow \mathcal{X}$ the stabilizer group $G_{\zeta}$ is a closed subgroup scheme of $G L_{n, k}$ compatibly with the composite map

$$
B G_{\zeta} \stackrel{\widetilde{\zeta}}{\rightarrow} \mathcal{X} \rightarrow B G L_{n}
$$

Of course every representation of $G L_{n}$ over $k$ induces a representation of $G_{\zeta}$, i.e. every quasi-coherent sheaf on $B G L_{n, k}$ pulls back to a quasi-coherent

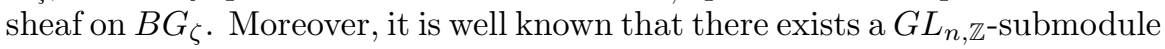

$$
W \subset H^{0}\left(G L_{n, \mathbb{Z}}, \mathcal{O}_{G L_{n, \mathbb{Z}}}\right)
$$

which is a finitely-generated free $\mathbb{Z}$-module such that the composite map

$$
W \otimes_{\mathbb{Z}} k \rightarrow H^{0}\left(G L_{n, k}, \mathcal{O}_{G L_{n, k}}\right) \rightarrow H^{0}\left(G_{\zeta}, \mathcal{O}_{G_{\zeta}}\right)
$$

is surjective. So for each point $\zeta:$ Spec $k \rightarrow \mathcal{X}$, there is a $G L_{n, \mathbb{Z}}$ representation $W$ which is a finitely-generated free $\mathbb{Z}$-module such that the pullback $\mathcal{E}$ under $\mathcal{X} \rightarrow B G L_{n, \mathbb{Z}}$ of the locally free sheaf associated to $W$ satisfies the condition in theorem 5.2. In order to apply theorem 5.2. we need to find a single representation $W$ of $G L_{n, \mathbb{Z}}$ which works for every geometric point $\zeta:$ Spec $k \rightarrow \mathcal{X}$.

(3) The stack $B G_{\zeta}$ is naturally isomorphic to the fiber product

$$
\mathcal{X} \times_{X, \pi \circ \zeta} \operatorname{Spec} k
$$

and the map $\widetilde{\zeta}$ is simply the projection onto the first factor.

We will prove theorem 5.2 after proving a proposition about quotient stacks by a finite group. Suppose that $\mathcal{E}$ is a locally free sheaf on $\mathcal{X}$ which satisfies the hypotheses of theorem 5.2 and suppose that $\mathcal{F}$ is a quasi-coherent sheaf on $\mathcal{X}$. To show that $\mathcal{E}$ is a generator for $\mathcal{F}$, it suffices to prove this after performing a faithfully-flat base change of the coarse moduli space $X$. By 1 , lemma 2.2.3], there is an étale cover $\left(X_{\alpha} \rightarrow X\right)$ such that for each $\alpha$ the fiber product $\mathcal{X} \times_{X} X_{\alpha}$ is of the form $[Y / G]$ where $Y$ is a finite scheme over $X_{\alpha}$ and $G$ is a finite group which acts on $Y$ via $X_{\alpha}$-morphisms. Moreover, since $\mathcal{X}$ is tame, we may assume that the order of $G$ is prime to the characteristic of every residue field of $X_{\alpha}$. So the first step in proving theorem 5.2 is the case that $\mathcal{X}=[Y / G]$ as above.

Now there is a map $f:[Y / G] \rightarrow B G$. Let $E$ denote the locally free sheaf on $B G$ corresponding to the left regular representation of $G$. If $\mathcal{E}$ is a locally free sheaf on $\mathcal{X}$ which is a generator for every quasi-coherent sheaf, then in particular it is a generator for $f^{*} E$. The following proposition shows that 
this is a sufficient condition for $\mathcal{E}$ to be a generator for every quasi-coherent sheaf.

Proposition 5.3. Suppose that $\mathcal{X}$ is a tame, separated Deligne-Mumford stack of the form $\mathcal{X}=[Y / G]$, as above. Let $f: \mathcal{X} \rightarrow B G$ and $E$ be as above. Then $f^{*} E$ is a generator for every quasi-coherent $\mathcal{O}_{\mathcal{X}}$-module $\mathcal{F}$.

Proof. Let $g: Y \rightarrow \mathcal{X}$ denote the quotient 1-morphism, and let $p: \mathcal{X} \rightarrow X$ denote the map to the coarse moduli space of $\mathcal{X}$. Observe that $f^{*} E$ is simply $g_{*} \mathcal{O}_{Y}$. The composition $p \circ g: Y \rightarrow X$ is a finite, surjective morphism of algebraic spaces. In particular, it is affine and for each quasi-coherent $\mathcal{O}_{Y^{-}}$ module $\mathcal{G}$, the induced morphism

$$
\alpha:(p \circ g)^{*}(p \circ g)_{*} \mathcal{G} \rightarrow \mathcal{G},
$$

is surjective. By adjointness of $p_{*}$ and $p^{*}$, there is an induced morphism

$$
p^{*}(p \circ g)_{*} \mathcal{G} \rightarrow g_{*} \mathcal{G},
$$

in fact this is precisely $\theta_{\mathcal{O}_{\mathcal{X}}}\left(g_{*} \mathcal{G}\right)$. Since $g_{*} \mathcal{G}$ is a module over $g_{*} \mathcal{O}_{Y}$, we get an induced morphism of $\mathcal{O}_{\mathcal{X}}$-modules

$$
\phi: g_{*} \mathcal{O}_{Y} \otimes_{\mathcal{O}_{\mathcal{X}}} p^{*}(p \circ g)_{*} \mathcal{G} \rightarrow g_{*} \mathcal{G} .
$$

The claim is that $\phi$ is surjective; let us assume this for a moment. The canonical injection $g^{\#}: \mathcal{O}_{\mathcal{X}} \rightarrow g_{*} \mathcal{O}_{Y}$ induces a morphism of $\mathcal{O}_{\mathcal{X}}$-modules

$\psi: g_{*} \mathcal{O}_{Y} \otimes_{\mathcal{O}_{\mathcal{X}}} p^{*} p_{*} \operatorname{Hom}_{\mathcal{O}_{\mathcal{X}}}\left(g_{*} \mathcal{O}_{Y}, g_{*} \mathcal{G}\right) \rightarrow g_{*} \mathcal{O}_{Y} \otimes_{\mathcal{O}_{\mathcal{X}}} p^{*} p_{*} H_{o m} m_{\mathcal{X}}\left(\mathcal{O}_{\mathcal{X}}, g_{*} \mathcal{G}\right)$.

Since $g$ is representable, finite and flat, there is a surjective trace map

$$
t: g_{*} \mathcal{O}_{Y} \rightarrow \mathcal{O}_{\mathcal{X}}
$$

which splits the injection $g^{\#}$. Therefore $\psi$ is surjective. And $\theta_{\mathcal{E}}\left(g_{*} \mathcal{G}\right)$ is the composite $\phi \circ \psi$. Since $\psi$ and $\phi$ are both surjective, we conclude that $\theta_{\mathcal{E}}\left(g_{*} \mathcal{G}\right)$ is surjective. So to prove that $\theta_{\mathcal{E}}\left(g_{*} \mathcal{G}\right)$ is surjective, it suffice to prove the claim that $\phi$ is surjective.

To see that $\phi$ is surjective, we apply $g^{*}$; since $g$ is faithfully flat, we may check surjectivity after base-change by $g$. Since $g$ is affine, the canonical morphism

$$
\left(g^{*} g_{*} \mathcal{O}_{Y}\right) \otimes_{\mathcal{O}_{Y}} \mathcal{G} \rightarrow g^{*} g_{*} \mathcal{G}
$$

is an isomorphism. Similarly, the canonical morphism

$$
\left(g^{*} g_{*} \mathcal{O}_{Y}\right) \otimes_{\mathcal{O}_{Y}}(p \circ g)^{*}(p \circ g)_{*} \mathcal{G} \rightarrow g^{*}\left(g_{*} \mathcal{O}_{Y} \otimes_{\mathcal{O}_{\mathcal{X}}} p^{*}(p \circ g)_{*} \mathcal{G}\right)
$$

is also an isomorphism. And we have a commutative diagram

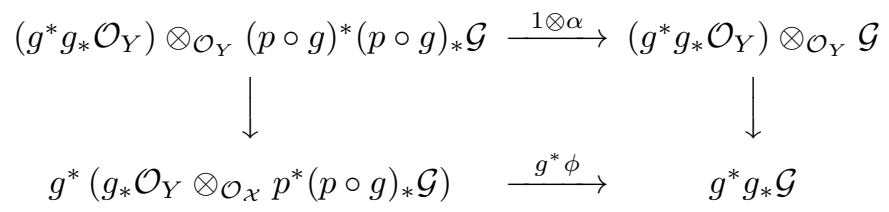


Since $\alpha$ is surjective, so is $1 \otimes \alpha$. Therefore $g^{*} \phi$ is surjective, and it follows that $\phi$ is surjective. This proves the claim, and we conclude that $f^{*} E$ is a generator for all sheaves of the form $g_{*} \mathcal{G}$ with $\mathcal{G}$ a quasi-coherent $\mathcal{O}_{Y}$-module.

Let $\mathcal{F}$ be a quasi-coherent $\mathcal{O}_{\mathcal{X}}$-module. Since $g$ is affine, the canonical morphism of $\mathcal{O}_{\mathcal{X}}$-modules

$$
g_{*} \mathcal{O}_{Y} \otimes_{\mathcal{O}_{\mathcal{X}}} \mathcal{F} \rightarrow g_{*} g^{*} \mathcal{F},
$$

is an isomorphism. Therefore $f^{*} E$ is a generator for $g_{*} \mathcal{O}_{Y} \otimes_{\mathcal{O}_{\mathcal{X}}} \mathcal{F}$. And we have a surjective morphism of $\mathcal{O}_{\mathcal{X}}$-modules,

$$
t \otimes 1: g_{*} \mathcal{O}_{Y} \otimes_{\mathcal{O}_{\mathcal{X}}} \mathcal{F} \rightarrow \mathcal{F} .
$$

Since $G_{f^{*} E}$ is a right-exact functor, we conclude that $f^{*} E$ is also a generator for $\mathcal{F}$.

Corollary 5.4. Suppose that $\mathcal{X}, f: \mathcal{X} \rightarrow B G$, and $E$ are as in proposition 5.3. If $\mathcal{E}$ is a locally free sheaf on $\mathcal{X}$ which generates $f^{*} E$, then $\mathcal{E}$ generates $\mathcal{F}$ for every quasi-coherent $\mathcal{O}_{\mathcal{X}}$-module $\mathcal{F}$.

Proof. Since $p_{*}$ is exact, for any quasi-coherent $\mathcal{O}_{X}$-module $\mathcal{G}$ and any quasicoherent $\mathcal{O}_{\mathcal{X}}$-module $\mathcal{F}$, we have that

$$
p_{*}\left(p^{*} \mathcal{G} \otimes_{\mathcal{O}_{\mathcal{X}}} \mathcal{F}\right)=\mathcal{G} \otimes_{\mathcal{O}_{X}} p_{*} \mathcal{F} .
$$

To see this, it suffices to work locally over affine opens in $X$, so we may suppose that $\mathcal{G}$ is a colimit of finitely-presented $\mathcal{O}_{X}$-modules. Since $p_{*}$ commutes with colimits, we are reduced to the case that $\mathcal{G}$ is finitely-presented. Since $p^{*}$ is right-exact and since $p_{*}$ is exact, we are reduced to the case that $\mathcal{G}=\mathcal{O}_{X}$, which is trivial.

Similarly, we conclude that $\theta_{\mathcal{E}}\left(p^{*} \mathcal{G} \otimes_{\mathcal{O}_{\mathcal{X}}} \mathcal{F}\right)$ is just $1_{p^{*} \mathcal{G}} \otimes \theta_{\mathcal{E}}(\mathcal{F})$. In particular, if $\mathcal{E}$ generates $\mathcal{F}$, then $\mathcal{E}$ generates $p^{*} \mathcal{G} \otimes_{\mathcal{O}_{\mathcal{X}}} \mathcal{F}$. Therefore, $\mathcal{E}$ generates every sheaf of the form $p^{*} \mathcal{G} \otimes_{\mathcal{O}_{\mathcal{X}}} f^{*} E$. But by proposition [5.3, we conclude that every quasi-coherent sheaf $\mathcal{F}$ is a surjective image of a sheaf of the form $p^{*} \mathcal{G} \otimes_{\mathcal{O}_{\mathcal{X}}} f^{*} E$. Since $G_{\mathcal{E}}$ is right-exact, we conclude that $\mathcal{E}$ generates $\mathcal{F}$.

Now we come to the proof of theorem 5.2 .

Proof. Suppose $\mathcal{E}$ satisfies the hypotheses of theorem [5.2. By [1, lemma 2.2.3], there is an étale morphism $g: X^{\prime} \rightarrow X$ whose image contains the image of $\zeta($ Spec $k)$ and such that $\mathcal{X}^{\prime}=\mathcal{X} \times_{X} X^{\prime}$ is of the form $[Y / G]$ for some finite $X^{\prime}$-scheme $Y$, for some finite group $G$ and an action of $G$ on $Y$ via $X^{\prime}$-morphisms. Let $f:[Y / G] \rightarrow B G$ and $E$ be as in proposition 5.3 , Let $\mathcal{E}^{\prime}$ denote the pullback of $\mathcal{E}$ to $\mathcal{X}^{\prime}$. Consider the morphism $\theta_{\mathcal{E}^{\prime}}\left(f^{*} E\right)$. Denote the cokernel of this morphism by $\mathcal{G}$. Then $\mathcal{G}$ is a finitely-generated quasi-coherent sheaf, and therefore has closed support $Z \subset \mathcal{X}$.

Since $k$ is algebraically-closed and since $X^{\prime} \rightarrow X$ is finite, we can find a factorization $\xi$ : Spec $k \rightarrow X^{\prime}$ of Spec $k \rightarrow X$. Now the functors $F$ and $G$ 
and the natural transformation $\theta$ are compatible with arbitrary base change on $X$. Therefore the pullback of $\theta_{\mathcal{E}^{\prime}}\left(f^{*} E\right)$ equals $\theta_{\xi^{*} \mathcal{E}^{\prime}}\left(\xi^{*} f^{*} E\right)$. Of course we have

$$
\mathcal{X}^{\prime} \times_{X^{\prime}} \text { Spec } k=\mathcal{X} \times_{X} \text { Spec } k=B G_{\zeta} .
$$

By hypothesis $\xi^{*} \mathcal{E}^{\prime}=\widetilde{\zeta}(\mathcal{E})$ generates the regular representation of $G_{\zeta}$. So by corollary 5.4 we conclude that $\theta_{\xi^{*} \mathcal{E}^{\prime}}\left(\xi^{*} f^{*} E\right)$ is surjective. So the image of $\xi($ Spec $k)$ is not in the support of $\mathcal{G}$. Denoting by $V \subset X^{\prime}$ the complement of the image of the support of $\mathcal{G}$, we have that $V$ contains $\xi$ (Spec $k$ ) and the restriction of $\mathcal{E}^{\prime}$ over $V$ generates $f^{*} E$.

Let $U$ be the image of $V \rightarrow X$ so that the canonical morphism $h: \mathcal{X} \times_{X}$ $V \rightarrow \mathcal{X} \times{ }_{X} U$ is faithfully flat and $U$ contains the image of $\zeta(\operatorname{Spec} k)$. Let $\mathcal{F}$ be any quasi-coherent sheaf on $\mathcal{X} \times{ }_{X} U$. Then $h^{*} \theta_{\mathcal{E}}(\mathcal{F})$ is $\theta_{h^{*} \mathcal{E}}\left(h^{*} \mathcal{F}\right)$. By corollary [5.4] we know that $\theta_{h^{*} \mathcal{E}}\left(h^{*} \mathcal{F}\right)$ is surjective. Since $h$ is faithfully flat, we conclude that also $\theta_{\mathcal{E}}(\mathcal{F})$ is surjective. So the restriction of $\mathcal{E}$ to $\mathcal{X} \times{ }_{X} U$ is a generator for every quasi-coherent sheaf $\mathcal{F}$ on $\mathcal{X} \times{ }_{X} U$. This completes the proof of the theorem.

Now we come to the main theorem of this section:

Theorem 5.5. Suppose that $\mathcal{X}$ is a quasi-compact, tame Deligne-Mumford stack of the form $\left[Y / G L_{n, \mathbb{Z}}\right]$ for some scheme $Y$ and an action of $G L_{n, \mathbb{Z}}$. Let $f: \mathcal{X} \rightarrow B G L_{n, \mathbb{Z}}$ be the obvious 1-morphism. There exists a finitelygenerated free $\mathbb{Z}$-module, $W$ which is a representation of $G L_{n, \mathbb{Z}}$ such that if $E$ on $B G L_{n, \mathbb{Z}}$ is the induced locally free sheaf then $f^{*} E$ has the following property: For every morphism of algebraic spaces $g: X^{\prime} \rightarrow X$ with corresponding map $h: \mathcal{X} \times_{X} X^{\prime} \rightarrow \mathcal{X}$ and for every quasi-coherent sheaf $\mathcal{F}$ on $\mathcal{X} \times_{X} X^{\prime}$, we have that $h^{*} f^{*} E$ is a generator for $\mathcal{F}$.

Proof. First of all suppose we prove the result when $g: X^{\prime} \rightarrow X$ is the identity map. Then by remark (1) following the statement of theorem 5.2. it follows that for every algebraically-closed field $k$ and every 1-morphism $\zeta:$ Spec $k \rightarrow \mathcal{X}$ we have that $\widetilde{\zeta}^{*} f^{*} E$ generates the left regular representation of $G_{\zeta}$. Now suppose that $g: X^{\prime} \rightarrow X$ is an arbitrary morphism, $k$ is an algebraically-closed field and $\xi$ : Spec $k \rightarrow \mathcal{X} \times_{X} X^{\prime}$ is a 1-morphism. Let $\zeta$ : Spec $k \rightarrow \mathcal{X}$ be $h \circ \xi$. Then $G_{\xi}=G_{\zeta}$, and $\xi^{*} h^{*} f^{*} E=\zeta^{*} f^{*} E$. So we see that $h^{*} f^{*} E$ on $\mathcal{X} \times_{X} X^{\prime}$ satisfies the hypotheses of theorem [5.2. So $h^{*} f^{*} E$ generates every quasi-coherent sheaf. Therefore to prove our theorem, we need only consider the case that $g: X^{\prime} \rightarrow X$ is the identity map.

By remark (2) following the statement of theorem 5.2, for each geometric point $\zeta$ : Spec $k \rightarrow \mathcal{X}$, there is a finitely-generated free $\mathbb{Z}$-module $W_{\zeta}$ which is a representation of $G L_{n, \mathbb{Z}}$ and such that if $E_{\zeta}$ is the corresponding locally free sheaf on $B G L_{n, \mathbb{Z}}$, then $\widetilde{\zeta}^{*} f^{*} W_{\zeta}$ generates the left regular representation. Then by theorem [5.2, there is a Zariski open neighborhood $U_{\zeta} \subset X$ of the image of $\zeta($ Spec $k)$ such that the restriction of $f^{*} E_{\zeta}$ to $\mathcal{X} \times_{X} U_{\zeta}$ generates all quasi-coherent sheaves. Allowing $k$ and $\zeta$ to vary over all algebraicallyclosed fields and 1-morphisms, we obtain a Zariski open cover $\left\{U_{\zeta}\right\}$ of $X$. 
Since $\mathcal{X}$ is quasi-compact, some finite subset covers $X$, say $\zeta_{1}, \ldots, \zeta_{N}$. Let

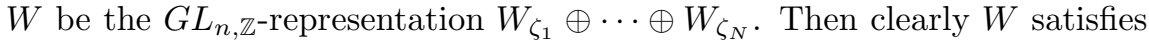
the conditions in the theorem.

\section{Natural transformation of Quot Functors}

Suppose that $S$ is a quasi-compact algebraic space, $f: \mathcal{X} \rightarrow S$ is a separated, finitely-presented 1-morphism of a tame Deligne-Mumford stack to $S$ such that $\mathcal{X}$ is a global quotient over $S$, say $\mathcal{X}=\left[Y / G L_{n, X}\right]$. By theorem [5.5. there is a $G L_{n, \mathbb{Z}}$ representation $V$ which is a finite free $\mathbb{Z}$ module and such that for every morphism of algebraic spaces $T \rightarrow S$ and every quasi-coherent sheaf $\mathcal{G}$ on $T \times_{S} \mathcal{X}, \mathcal{E}_{V}$ generates $\mathcal{G}$.

Suppose that $\mathcal{F}$ is a locally finitely-presented $\mathcal{O}_{\mathcal{X}}$-module. Suppose that $P: K^{0}(\mathcal{X}) \rightarrow \mathbb{Z}$ is a given Hilbert polynomial. Let $p: \mathcal{X} \rightarrow X$ denote the coarse moduli scheme of $\mathcal{X}$ and let $P_{V}: K^{0}(X) \rightarrow \mathbb{Z}$ denote the map

$$
P_{V}([E])=P\left(\left[H o m_{\mathcal{O}_{\mathcal{X}}}\left(\mathcal{E}_{V}, p^{*} E\right)\right]\right) .
$$

Suppose $T \rightarrow S$ is a morphism of a scheme to $S$ and suppose that $\mathcal{F}_{T} \rightarrow \mathcal{G}$ is an element of $Q^{P}(\mathcal{F} / \mathcal{X} / S)(T)$. Consider $F_{\mathcal{E}_{V}}\left(\mathcal{F}_{T}\right) \rightarrow F_{\mathcal{E}_{V}}(\mathcal{G})$. Since $F_{\mathcal{E}_{V}}$ is exact, this is still surjective. By lemma 4.1 $F_{\mathcal{E}_{V}}(\mathcal{G})$ is locally finitelypresented, has proper support over $T$ and is flat over $T$. Moreover every geometric fiber has Hilbert polynomial $P_{V}$, i.e. $F_{\mathcal{E}_{V}}\left(\mathcal{F}_{T}\right) \rightarrow F_{\mathcal{E}_{V}}(\mathcal{G})$ is an element of $Q^{P_{V}}\left(F_{\mathcal{E}_{V}} / X / S\right)(T)$. This defines a natural transformation

$$
F_{\mathcal{E}_{V}}: Q^{P}(\mathcal{F} / \mathcal{X} / S) \Rightarrow Q^{P_{V}}\left(F_{\mathcal{E}_{V}}(\mathcal{F}) / X / S\right)
$$

Lemma 6.1. The natural transformation $F_{\mathcal{E}_{V}}$ is a monomorphism, i.e. for each $T \rightarrow S$ the morphism of sets

$$
F_{\mathcal{E}_{V}}(T): Q^{P}(\mathcal{F} / \mathcal{X} / S)(T) \rightarrow Q^{P_{V}}\left(F_{\mathcal{E}_{V}}(\mathcal{F}) / X / S\right)(T),
$$

is an injection of sets.

Proof. Given any morphism $T \rightarrow S$ of a scheme to $S$ and given any element $\alpha: F_{\mathcal{E}_{V}}(\mathcal{F})_{T} \rightarrow G$ in $Q^{P_{V}}\left(F_{\mathcal{E}_{V}}(\mathcal{F}) / X / S\right)(T)$, define $\beta: K \rightarrow F_{\mathcal{E}_{V}}(\mathcal{F})_{T}$ to be the kernel of $\alpha$ and define $\eta_{T}(\alpha): \mathcal{F} \rightarrow \mathcal{G}$ to be the cokernel of the composition:

$$
p^{*} K \otimes_{\mathcal{O}_{\mathcal{X}}} \mathcal{E}_{V} \stackrel{p^{*} \beta \otimes 1}{\longrightarrow} G_{\mathcal{E}_{V}}\left(\mathcal{F}_{T}\right) \stackrel{\theta_{\mathcal{E}_{V}}\left(\mathcal{F}_{T}\right)}{\longrightarrow} \mathcal{F}_{T}
$$

Now suppose given $\nu: \mathcal{F}_{T} \rightarrow \mathcal{G}$ in $Q^{P}(\mathcal{F} / \mathcal{X} / S)(T)$ and let $\mu: \mathcal{K} \rightarrow \mathcal{F}_{T}$ denote the kernel of $\nu$. Then we have a short exact sequence:

$$
0 \longrightarrow F_{\mathcal{E}_{V}}(\mathcal{K}) \stackrel{F_{\mathcal{E}_{V}}(\mu)}{\longrightarrow} F_{\mathcal{E}_{V}}(\mathcal{F})_{T} \stackrel{F_{\mathcal{E}_{V}}(\nu)}{\longrightarrow} F_{\mathcal{E}_{V}}(\mathcal{G}) \longrightarrow 0 .
$$


Notice that if $\alpha=F_{\mathcal{E}_{V}}(\nu)$, then $\beta=F_{\mathcal{E}_{F}}(\mu)$. Since $p^{*}$ is right-exact, we have a commutative diagram of exact sequences:

$$
\begin{aligned}
& G_{\mathcal{E}_{V}}(\mathcal{K}) \stackrel{p^{*} \beta \otimes 1}{\longrightarrow} G_{\mathcal{E}_{V}}\left(\mathcal{F}_{T}\right) \stackrel{p^{*} \alpha \otimes 1}{\longrightarrow} G_{\mathcal{E}_{V}}(\mathcal{G}) \longrightarrow 0 \\
& \theta_{\mathcal{E}_{V}}(\mathcal{K}) \downarrow \quad \theta_{\mathcal{E}_{V}}\left(\mathcal{F}_{T}\right) \downarrow \quad \theta_{\mathcal{E}_{V}}(\mathcal{G}) \downarrow \\
& 0 \longrightarrow \mathcal{K} \stackrel{\mu}{\longrightarrow} \mathcal{E}_{T} \stackrel{\nu}{\longrightarrow} \mathcal{K} \longrightarrow 0
\end{aligned}
$$

It follows by the snake lemma that we have the formula:

$$
\eta_{T}\left(F_{\mathcal{E}_{V}}(\mu)\right)=\mu \text {. }
$$

Therefore $F_{\mathcal{E}_{V}}$ is injective.

Remark: Notice that the association $T \mapsto \eta_{T}$ is functorial for arbitrary $S$-morphisms $T_{1} \rightarrow T_{2}$. This might seem odd since the formation of the kernel $K$ is not compatible arbitrary base-change. But one could define $\eta_{T}$ equivalently to be the coequalizer of the diagram:

$$
\begin{aligned}
& G_{\mathcal{E}_{V}}\left(\mathcal{F}_{T}\right) \stackrel{p^{*} \alpha \otimes 1}{\longrightarrow} p^{*} G \otimes \mathcal{O}_{\mathcal{X}} \mathcal{E}_{V} \\
& \theta_{\mathcal{E}_{V}}\left(\mathcal{F}_{T}\right) \downarrow \\
& \quad \mathcal{F}_{T}
\end{aligned}
$$

Since coequalizers are compatible with arbitrary base-change, we see that $\eta$ is compatible with arbitrary $S$-morphisms $T_{1} \rightarrow T_{2}$.

Proposition 6.2. The monomorphism $F_{\mathcal{E}_{V}}$ of functors is relatively representable by schemes. In fact, $F_{\mathcal{E}_{V}}$ is a finitely-presented, quasi-finite, monomorphism. If $\mathcal{F}$ has proper support over $S$, then $F_{\mathcal{E}_{V}}$ is a finitelypresented, finite, monomorphism, i.e. a finitely-presented closed immersion.

Proof. Suppose $T \rightarrow S$ is a morphism of a scheme to $S$ and suppose that $\alpha: F_{\mathcal{E}_{V}}\left(\mathcal{F}_{T}\right) \rightarrow G$ is an element of $Q^{P_{V}}\left(F_{\mathcal{E}_{V}}(\mathcal{F}) / X / S\right)$. Now form $\eta_{T}(\alpha)$ : $\mathcal{F}_{T} \rightarrow \mathcal{G}$. By theorem 3.2, there is a flattening stratification $g: \Sigma \rightarrow T$ for $\mathcal{G}$ and $g$ is a surjective, finitely-presented, quasi-finite monomorphism. By lemma 4.3, for each connected component $\Sigma_{i}$ of $\Sigma$, the restriction of $\mathcal{G}$ to $\Sigma_{i}$ has constant Hilbert polynomial $P_{i}$. In particular, there is a connected component (possibly empty) $\Sigma_{i}$ of $\Sigma$ on which the restriction of $\mathcal{G}$ has Hilbert polynomial $P$. Of course we have an induced natural transformation of functors

$$
\Sigma_{i} \rightarrow T \times{ }_{\alpha, Q^{P} V\left(F_{\mathcal{E}_{V}}(\mathcal{F}) / X / S\right)} Q^{P}(\mathcal{F} / \mathcal{X} / S) .
$$

By equation 49, we have an inverse natural transformation. Thus we conclude that the fiber functor $T \times_{\alpha, Q^{P_{V}}\left(F_{\mathcal{E}_{V}}(\mathcal{F}) / X / S\right)} Q^{P}(\mathcal{F} / \mathcal{X} / S)$ is represented by the morphism $g_{i}: \Sigma_{i} \rightarrow T$. Notice that this is a finitely-presented, quasifinite monomorphism of schemes. 
In case $\mathcal{F}$ has proper support over $S$, we know from theorem 1.1 that $Q^{P} \rightarrow S$ satisfies the valuative criterion of properness. Therefore $g_{i}: \Sigma_{i} \rightarrow T$ satisfies the valuative criterion of properness, i.e. $g_{i}$ is finite. But a finite monomorphism is precisely a closed immersion, therefore $g_{i}$ is a finitelypresented closed immersion.

Now we are in a position to prove theorem 4.4 By proposition 6.2 we know that

$$
Q^{P}(\mathcal{F} / \mathcal{X} / S) \rightarrow Q^{P_{V}}\left(F_{\mathcal{E}_{V}}(\mathcal{F}) / X / S\right)
$$

is relatively representable by a finitely-presented, quasi-finite monomorphism (resp. finitely-presented closed immersion).

Now the proof that $Q^{P_{V}}\left(F_{\mathcal{E}_{V}}(\mathcal{F}) / X / S\right) \rightarrow S$ is represented by a scheme which is quasi-projective over $S$ is essetially [6, théorème 3.2, part IV]. In his proof, Grothendieck makes some Noetherian hypotheses which are eliminated in a standard way, cf. [4, section IV.8.9]. For the sake of completeness, we include the proof here.

We have a locally closed immersion $X \hookrightarrow \mathbb{P}_{S}^{N}$ for some $N$. By [4, proposition IV.8.9.1], we can find a finite-type affine $\mathbb{Z}$-scheme, $S^{\prime}$, a quasi-projective (resp. projective) $S^{\prime}$-scheme, $X^{\prime}$, and a coherent sheaf $F^{\prime}$ on $X^{\prime}$ along with a morphism $S \rightarrow S^{\prime}$ such that $X$ is isomorphic to $S \times{ }_{S^{\prime}} X^{\prime}$, and under this isomorphism $F_{\mathcal{E}_{V}}(\mathcal{F})$ is isomorphic to the pullback of $F^{\prime}$. Let $p: \mathbb{Z}\left[t, t^{-1}\right] \rightarrow \mathbb{Z}$ be the polynomial $p\left(t^{n}\right)=P_{V}\left(\left[\mathcal{O}_{X}(n)\right]\right)$ where $\mathcal{O}_{X}(n)$ is the pullback to $X$ of the invertible sheaf $\mathcal{O}_{\mathbb{P}_{S}^{N}}(n)$ on $\mathbb{P}_{S}^{N}$. polynomial, and consider the Quot functor $Q^{p}\left(F^{\prime} / X^{\prime} / S^{\prime}\right)$ which is the connected component of $Q\left(F^{\prime} / X^{\prime} / S^{\prime}\right)$ parametrizing families of quotients $F_{T}^{\prime} \rightarrow G$ such that for each closed point $t \in T$, we have $\chi\left(G_{t} \otimes \mathcal{O}(n)\right)=p\left(t^{n}\right)$. By lemma 4.3. it follows that $Q^{P_{V}}\left(F_{\mathcal{E}_{V}}(\mathcal{F}) / X / S\right)$ is isomorphic to a connected component of the fiber product $S \times \times_{S^{\prime}} Q^{p}\left(F^{\prime} / X^{\prime} / S^{\prime}\right)$. So to prove that $Q^{P_{V}}\left(F_{\mathcal{E}_{V}}(\mathcal{F}) / X / S\right) \rightarrow S$ is quasi-projective, it suffices to show that $Q^{p}\left(F^{\prime} / X^{\prime} / S^{\prime}\right) \rightarrow S^{\prime}$ is quasiprojective.

By [6, théorème 3.2, part IV], we know that the restriction of the functor $Q^{p}\left(F^{\prime} / X^{\prime} / S^{\prime}\right)$ to the category of locally Noetherian $S^{\prime}$-schemes is represented by a quasi-projective $S^{\prime}$-scheme. Using [4, proposition IV.8.9.1] and [4, théorème IV.11.2.6], it follows that in fact this quasi-projective $S^{\prime}$ scheme represents $Q^{p}\left(F^{\prime} / X^{\prime} / S^{\prime}\right)$ on the category of all $S^{\prime}$-schemes. Thus $Q^{P_{V}}\left(F_{\mathcal{E}_{V}}(\mathcal{F}) / X / S\right) \rightarrow S$ is represented by a quasi-projective scheme. This completes the proof of theorem 4.4.

\section{REFERENCES}

[1] D. Abramovich and A. Vistoli. Compactifying the space of stable maps. J. Amer. Math. Soc., 15(1):27-75 (electronic), 2002. 
[2] M. Artin. Algebraization of formal moduli. I. In Global Analysis (Papers in Honor of K. Kodaira), pages 21-71. Univ. Tokyo Press, Tokyo, 1969.

[3] S. Bosch, W. Lütkebohmert, and M. Raynaud. Néron models, volume 21 of Ergebnisse der Mathematik und ihrer Grenzgebiete (3) [Results in Mathematics and Related Areas (3)]. Springer-Verlag, Berlin, 1990.

[4] J. Dieudonné and A. Grothendieck. Éléments de géométrie algébrique. Inst. Hautes Études Sci. Publ. Math., 4, 8, 11, 17, 20, 24, 28, 32, 1961-1967.

[5] D. Edidin, B. Hassett, A. Kresch, and A. Vistoli. Brauer groups and quotient stacks. Amer. J. Math., 123(4):761-777, 2001.

[6] A. Grothendieck. Fondements de la géométrie algébrique. [Extraits du Séminaire Bourbaki, 1957-1962.]. Secrétariat mathématique, Paris, 1962.

[7] S. Keel and S. Mori. Quotients by groupoids. Ann. of Math. (2), 145(1):193-213, 1997.

[8] D. Knutson. Algebraic spaces. Springer-Verlag, Berlin, 1971. Lecture Notes in Mathematics, Vol. 203.

[9] G. Laumon and L. Moret-Bailly. Champs algébriques, volume 39 of Ergebnisse der Mathematik und ihrer Grenzgebiete. 3. Folge. A Series of Modern Surveys in Mathematics [Results in Mathematics and Related Areas. 3rd Series. A Series of Modern Surveys in Mathematics]. Springer-Verlag, Berlin, 2000.

[10] D. Mumford. Lectures on curves on an algebraic surface. With a section by G. M. Bergman. Annals of Mathematics Studies, No. 59. Princeton University Press, Princeton, N.J., 1966.

[11] M. Schlessinger. Functors of Artin rings. Trans. Amer. Math. Soc., 130:208-222, 1968. 